\title{
Rhinosinusal mucormycosis: Literature review and some particular cases
}

\author{
Carmen Badea ${ }^{1}$, Codrut Sarafoleanu ${ }^{1,2,3}$, Andreea Marza' \\ ${ }^{1}$ ENT\&HNS Department, "Sfanta Maria” Hospital, Bucharest, Romania \\ 2"Carol Davila" University of Medicine and Pharmacy, Bucharest, Romania \\ ${ }^{3}$ Center of excellence for research of sensorial and sensitive disorders, study of infecto-inflammatory, \\ tumoral and obstructive aero-digestive pathology (CESITO), ENT\&HNS Department, "Sfanta Maria" \\ Hospital, Bucharest, Romania
}

\begin{abstract}
Rhinosinusal mucormycosis is a life-threatening disease caused by fungus of the order Mucorales, which commonly affects individuals with diabetes and those in immunocompromised states. It is the most common form of mucormycosis with a high mortality rate $(50-80 \%)$. Treatment options include reversal of the underlying risk factors when it is possible, systemic antifungal medication and radical surgical debridement. Prognosis is reserved because of the high potential of invasiveness, so diagnosis and early treatment are essential.

Herein, we make a review about the most important features of this pathology and we report two cases of rhinosinusal mucormycosis with similar presentations who followed the same treatment protocol - extended surgical debridement of the necrotic tissue combined with systemic antifungal treatment (Amphotericin B). Complete recovery was achieved in one patient, whereas in the other one, due to late presentation, massive extension and incomplete surgical debridement, the disease was complicated with multiple organ dysfunction and cerebral stroke.

By presenting these cases, we would like to point out the importance of early diagnosis, appropriate medical and surgical therapy to obtain a significant survival rate in patients with this fatal disease.
\end{abstract}

KEYWORDS: mucormycosis, invasive fungal sinusitis, immunosuppression, Amphotericin B.

\section{INTRODUCTION}

Paltauf was the first one who described a case of rhinocerebral mucormycosis, in 1885, as "Mycosis Mucorina", but the disease name "mucormycosis" was given by an American pathologist named R.D. Baker to emphasize the etiologic agent ${ }^{1}$. Mucormycosis is an acute, aggressive, opportunistic infection caused by saprophytic fungus of the order Mucorales, with a high mortality and morbidity rates $(50-80 \%)$, which commonly affects individuals in immunocompromised states. The most common species involved are Rhizopus, Lichtheimia and Mucor $^{2}$.

Based on the clinical presentation, mucormycosis can be classified in 6 forms: rhino-orbito-cere- bral, pulmonary, gastrointestinal, cutaneous, renal and disseminated ${ }^{3}$.

Rhinosinusal mucormycosis is the most common form of the disease, found especially in patients with poorly-controlled diabetes mellitus ${ }^{4}$. Other underlying conditions are: patients with haematological malignancies, those with immunosuppression after organ transplantation, chronic use of corticosteroids or immunosuppressive agents, neutropenia, leukemias, lymphomas, high iron concentration, major burns, deferoxamine therapy, chronic renal failure. Rhinosinusal mucormycosis is often associated with corticosteroids used for graft-versus-host disease ${ }^{5,6}$.

Rhinosinusal mucormycosis develops in three 
stages. The first one is characterized by inflammation of the nasal mucosa and sinuses after inhalation of fungal spores. The second stage is characterized by orbital involvement and it manifests through the orbital apex syndrome (invasion of the optic nerve and the cranial nerves which control the eye movements - III, IV, VI and the ophthalmic division of the cranial nerve $\mathrm{V}$ ) and the sphenoid fissure syndrome (which affects cranial nerves III, IV, VI and V1 without optic neuropathy). Stage three manifests through cerebral involvement via one of the following routes: superior orbital fissure, ophthalmic artery or cribriform plate ${ }^{7,8}$.

\section{CLINICAL AND PARACLINICAL FEATURES OF MUCORMYCOSIS}

Clinical manifestations are non-specific and include: non-ocular symptoms (unilateral nasal obstruction, purulent nasal discharge, fever, facial pain with swelling, facial anaesthesia, recurrent epistaxis, necrotic lesions of the nasal mucosa, turbinates or hard palate), ocular symptoms (proptosis, periorbital cellulitis, blurry vision, ophthalmoplegia, central retina artery occlusion), neurological manifestation (headache, lethargy, hemiplegia, stroke, decreased mental function $)^{2,6}$.

Both computed tomography scan and nuclear magnetic resonance are useful in establishing the extent of the disease. The CT scan shows mucosal thickening, fluid filling the sinuses, bone erosion and intraorbital involvement. The MRI provides a better view of the blood vessels involvement and intracranial extension. Some studies reported that the unilateral nasal inflammation may represent the earliest imaging evidence of rhinosinusal mucormycosis in immunocompromised patients. It has been shown to have a higher value than bone erosion or extrasinusal extension, which are very suggestive for an invasive fungal disease, but seen only late in the infection course $e^{6,9,10}$.

The diagnosis of rhinosinusal mucormycosis is based on clinical and paraclinical examinations and confirmed by cultures and biopsy.

\section{TREATMENT STRATEGIES FOR MUCORMYCOSIS}

The current management of rhinosinusal mucormycosis requires four essential steps: (1) early diagnosis, complete surgical debridement of the necrotic tissue, combined with (3) prompt systemic antifungal therapy (Amphotericin B) and (4) reversal of the underlying risk factors ${ }^{11}$. It has been shown that the key to improve prognosis of this rapidly progressive disease is early diagnosis ${ }^{12}$. There are two diagnostic criteria proposed for the diagnosis of invasive fungal sinusitis: (1) rhinosinusitis confirmed radiologically and (2) histopathological evidence of fungus hyphae in the nasal mucosa, submucosa, blood vessels or bones ${ }^{13}$.

Surgical treatment requires radical surgical debridement of the necrotic tissues until bleeding tissue is observed, combined with local irrigation and packing of all the affected areas with Amphotericin B. In order to indicate the surgical approach, the preoperative radiographic imaging is mandatory to establish the extent of the disease. In early stages of the disease, functional endoscopic sinus surgery (FESS) has excellent results. However, in advanced stages with extension to the brain, orbit, buccal space, temporal fossa, etc., classic surgical procedures are required to obtain an adequate debridement of all the affected tissues ${ }^{6,14}$.

Polyenes represents the first line of antifungal therapy. Amphotericin B (AmB) is the main choice for the primary treatment of mucormycosis. Lipid derivates of AmB (Amphotericin B lipid complexABLC and liposomal Amphotericin B- LAmB) are less nephrotoxic than AmB and can be safely used for a longer period. There are no data regarding the optimal dosages. The doses vary between 1-1.5 $\mathrm{mg} / \mathrm{kg} / 24 \mathrm{~h}$ for $\mathrm{AmB}$ and $5-7.5 \mathrm{mg} / \mathrm{kg} / 24 \mathrm{~h}$ for LAmB and ABLC. Recent studies showed a better efficacy of LAmB over ABLC for the treatment of rhinosinusal mucormycosis with cerebral involvement. Until recently, polyenes represented the only class of antifungals used in the treatment of mucormycosis, but the new therapeutic advances suggested other agents that seem to possess potent activity against these fungi $i^{6,11}$.

In a recent retrospective study, it has been proved that the combination of lipid formulations of AmB with caspofungin therapy may improve the outcome of the patients with rhino-orbital-cerebral mucormycosis compared to polyene monotherapy ${ }^{15}$.

Posaconazole and deferasirox are drugs used as salvage therapy for patients with intolerance to polyenes or refractory disease. Posaconazole has the advantage of being able to be used for months to years due its low nephrotoxicity, but it cannot be used as primary therapy for mucormycosis. On the other hand, the combination polyene-posaconazole therapy does not bring any benefits compared to Amphotericin B monotherapy ${ }^{11,15}$.

Adjunctive therapy may have an additional benefit to standard treatment for patients with persistent neutropenia until they recovery from immunosuppression and it includes recombinant cytokines (G-CSF- granulocyte colony-stimulating 

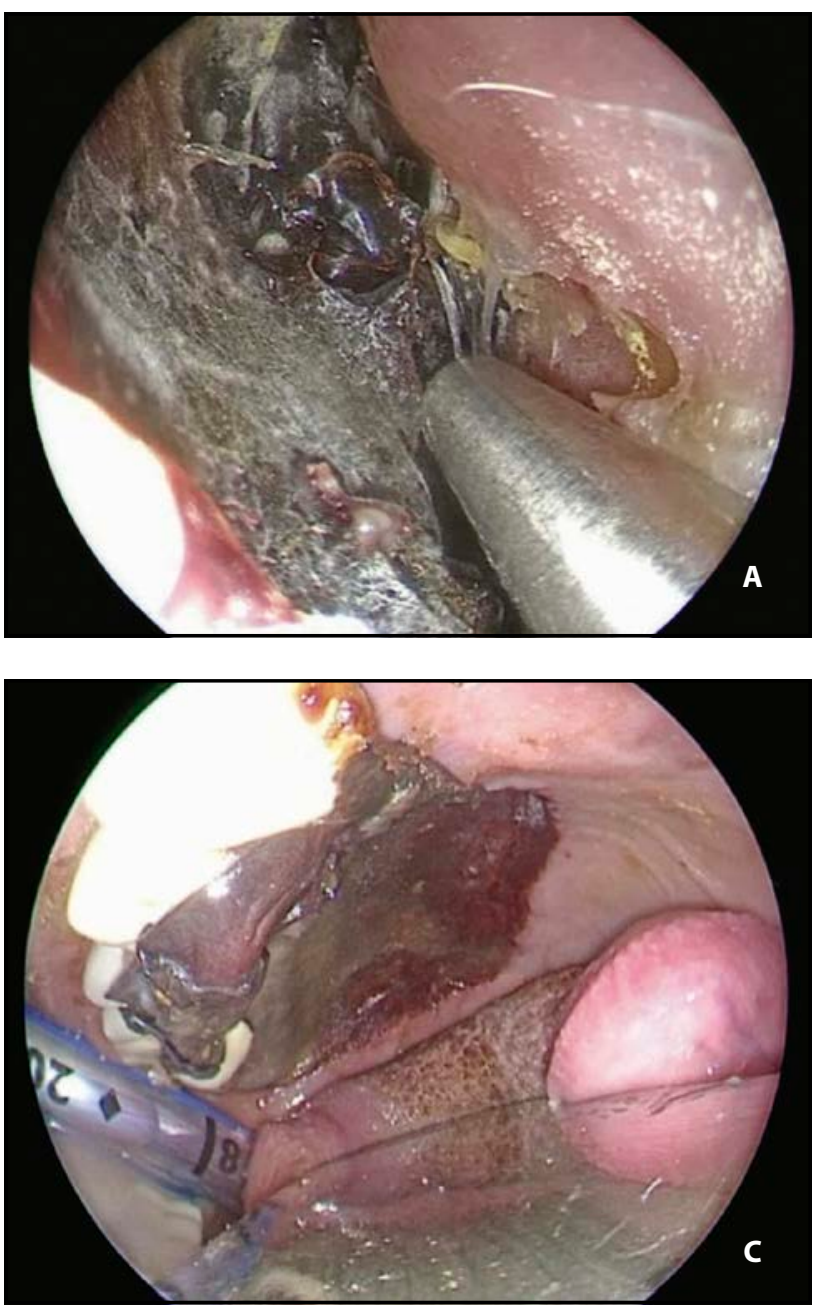

factor, GM-CSF-granulocyte macrophage colonystimulating factor and IFN $\gamma$ ), granulocyte transfusions and hyperbaric oxygen therapy ${ }^{15}$.

Nevertheless, no data exist to establish the total duration of therapy. The treatment should be individualized for each patient and it should be administered until certain objectives are achieved: resolution of radiological signs, clinical signs and symptoms of the disease and recovery from immunosuppression ${ }^{11}$.

In addition to medical and surgical treatment, the correction of the underlying risk factors, such as tapering of immunosuppressive medication, particularly corticosteroids, restoration of euglycemia and normal acid-base status is mandatory to achieve a better outcome ${ }^{6}$.

There are some obstacles that lead to difficulties in the management of mucormycosis. These include reduced accessibility to antifungal therapy, limitations of culture and molecular methods that delay initiation of antifungal therapy, nonspecific clinical manifestations which lead to misdiagnosis, lack of serological methods ${ }^{16}$.

In order to point out the importance of early diagnosis, appropriate medical and surgical ther-

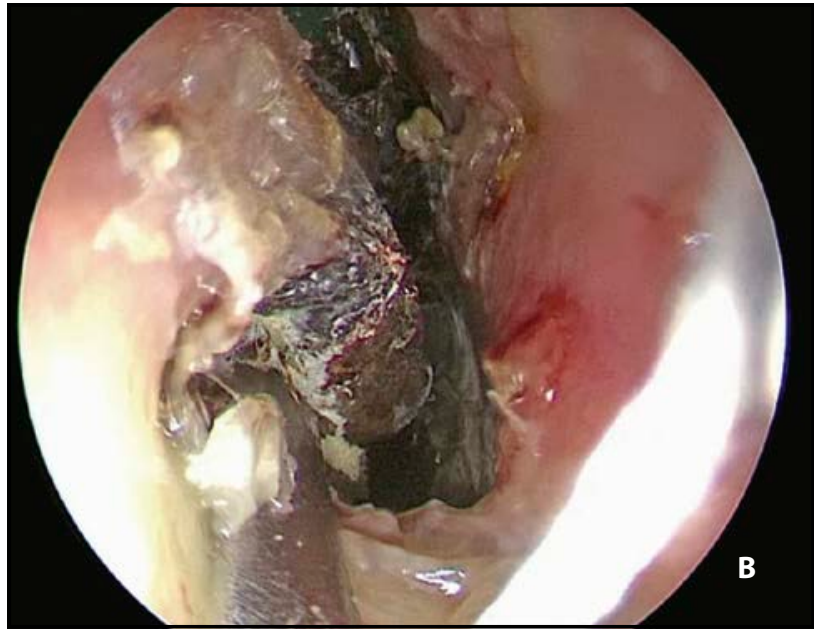

Figure 1 Intraoperative view: A,B. Necrosis of the middle and inferior turbinates and posterior portion of the nasal septum; C. Necrotic crust of the hard palate - macroscopic appearance suggestive of mucormycosis.

apy to obtain a significant survival rate in patients with this fatal disease, we present further two relevant cases.

\section{CASE 1}

A 54-year-old male patient presented to our ENT Clinic by transfer from another hospital unit with a chief complaint of severe headache for 1 month with subsequent development of hypoesthesia on the right side of the face, right periorbital oedema and palpebral ptosis. Medical history revealed poorly controlled type 2 diabetes mellitus and cirrhosis associated with chronic hepatitis $\mathrm{B}$ and $\mathrm{D}$ virus with liver transplantation 6 months before. He was treated with oral antidiabetics and immunosuppressive agents (tacrolimus and long-term corticotherapy).

The ENT clinical examination revealed necrotic areas and black ulceration on the hard palate, right nasal passage and the posterior portion of the nasal septum (Figure 1).

The laboratory tests at the time of admission revealed leucocytosis with 12.330 leucocytes, severe thrombocythemia (platelets 7000), renal dysfunction (serum creatinine $2.02 \mathrm{mg} / \mathrm{dl}$, BUN $272 \mathrm{mg} /$ dl), serum glucose level of $340.03 \mathrm{mg} / \mathrm{dl}$, fibrinogen $510 \mathrm{mg}$, CRP $202.80 \mathrm{mg}$, procalcitonin >=10. The culture of nasal discharge was positive for Aspergillus spp.

CT and MRI scan of the brain and paranasal sinuses revealed opacification of the right maxillary sinus, right ethmoidal cells and right sphenoidal sinus with extension into the orbit and bone erosion of the lateral and posterior wall of the right sphenoid sinus with cavernous sinus invasion (Figure 2).

Based on the history of uncontrolled diabetes, 

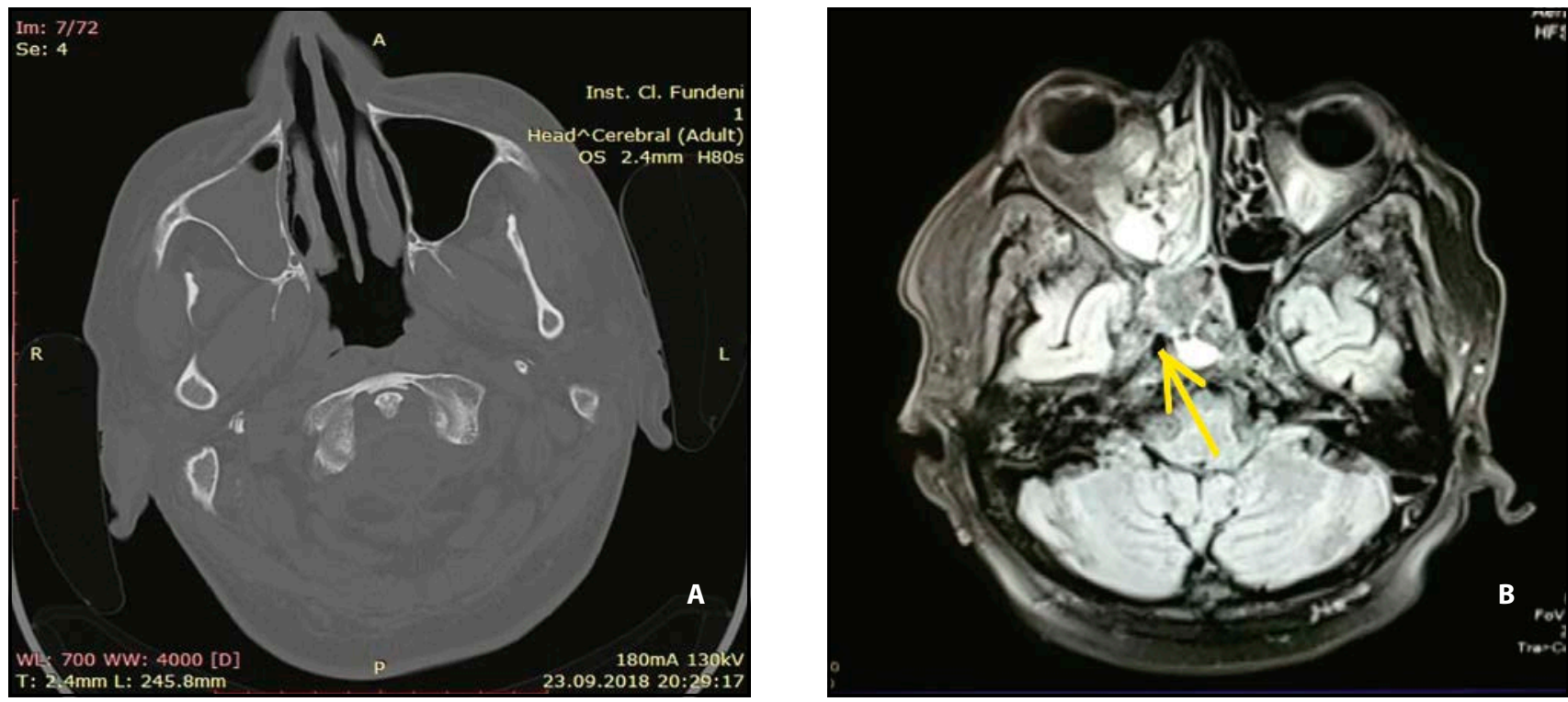

Figure 2 A. Cranio-facial CT scan, axial slice, showing opacification of the right maxillary sinus; B. T2-weighted axial MRI indicating hyperintensity of the front and rear right ethmoidal cells and right sphenoid sinus with orbital involvement; invasion of the right cavernous sinus (arrow).
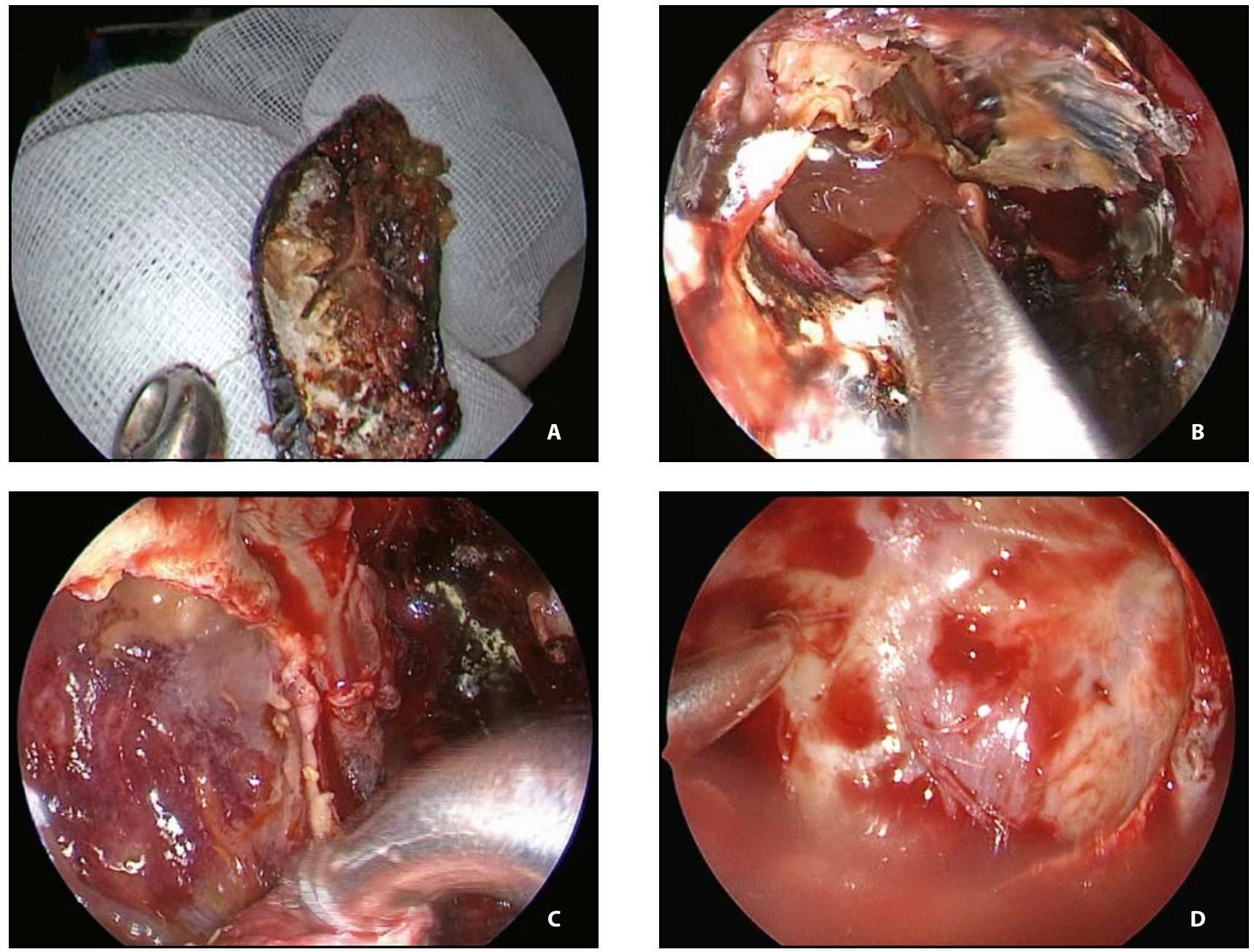

Figure 3 Intraoperative aspect-medial maxillectomy(A-D) A. The right inferior turbinate; B. Stagnant secretions in the right maxillary sinus; C. Right maxillary sinus mucosa- intraoperative view; D. Right maxillary sinus view post sinus mucosa ablation. 

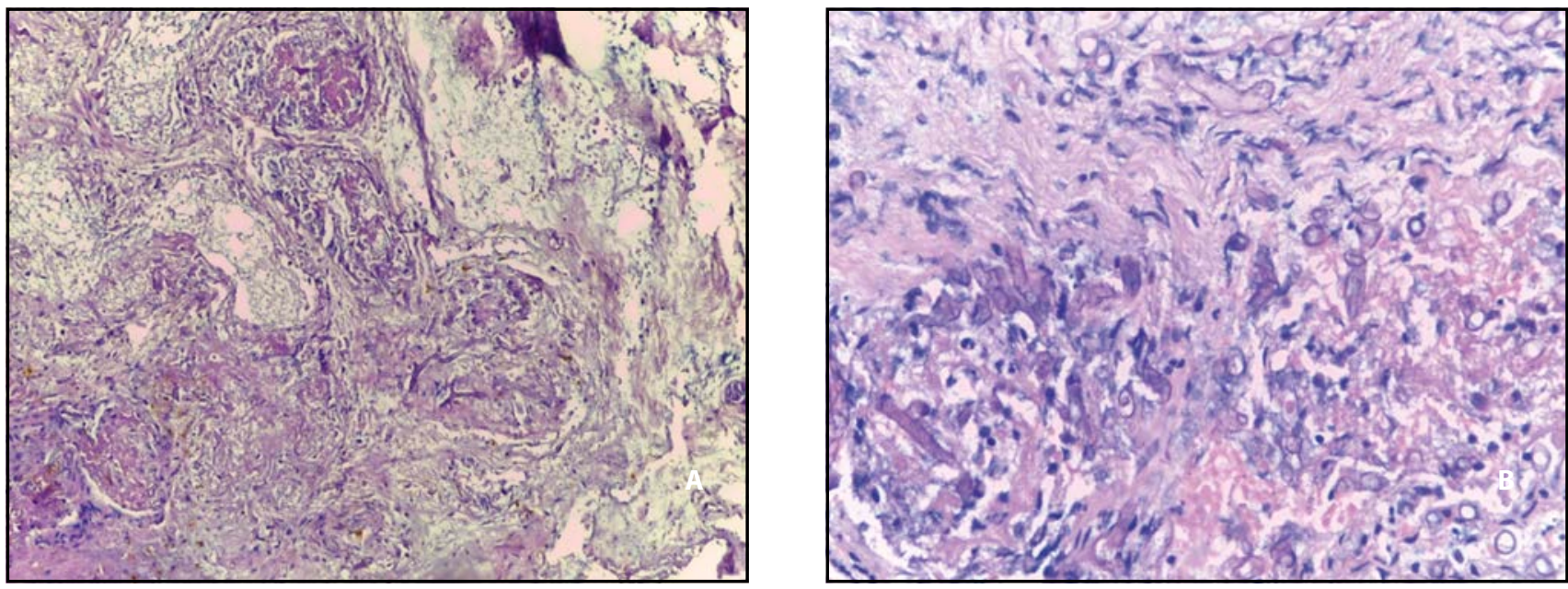

Figure 4 Histhopathology stains showing large, non-septate hyphae of Mucor and vascular invasion.

patient immunosuppression and clinical examination, a diagnosis of rhino-orbito-cerebral mucormycosis was considered.

The patient underwent aggressive endoscopic surgical debridement of necrotic areas of the inferior and middle turbinates, right medial maxillectomy and ethmoidectomy (Figure 3 ). The necrotic tissue was sent for anatomopathological examination and the right maxillary sinus secretion was sent for bacteriological examination. Histopathol-
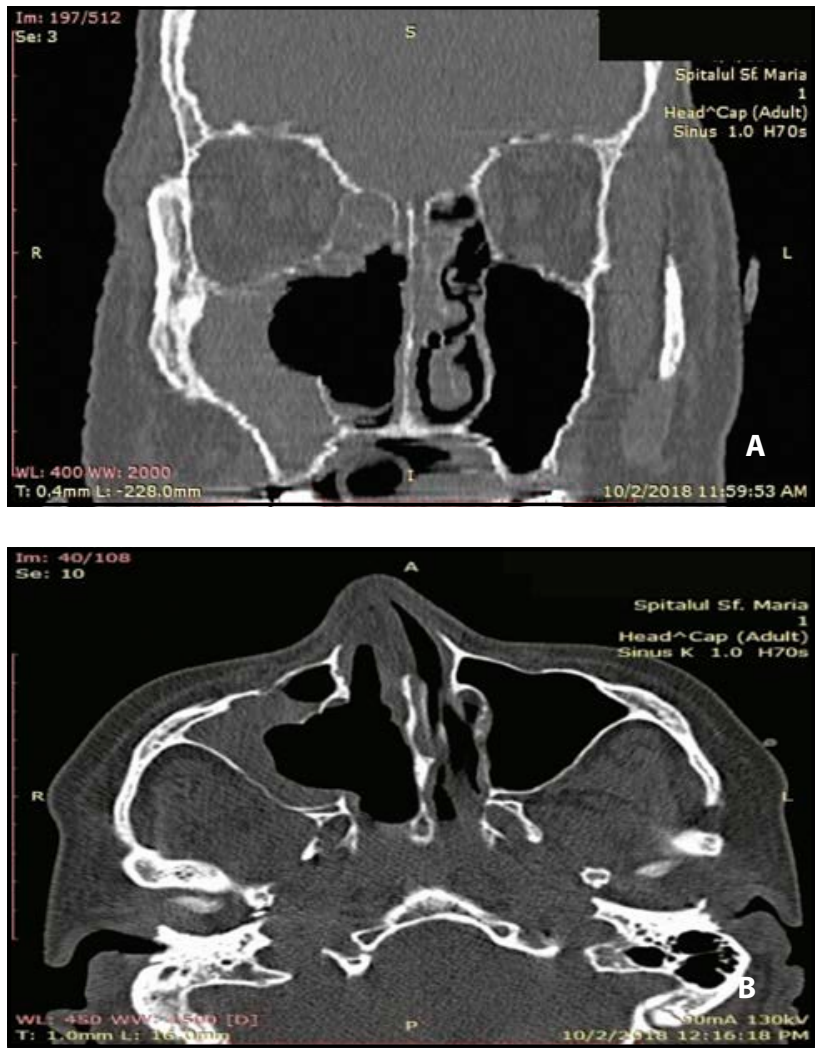

ogy of tissue stains showed nasal mucosa with haemorrhagic areas, areas of necrosis and the presence of hyphae suggestive for an invasive fungal rhinosinusitis - mucormycosis like (Figure 4). The culture of sinus secretion was positive for E. Coli, Pseudomonas spp and Enterococcus spp. The patient was subsequently treated with Amphotericin B $1.2 \mathrm{mg} / \mathrm{kg}$, antibiotics (Meronem, Linezolid, Metronidazole) and supportive treatment (platelet transfusions, erythrocyte mass, plasma, vitamin therapy, renal dialysis, hemofiltration).

Despite the aggressive therapy, the patient evolution was unfavourable. The CT scan was performed and confirmed the progression of the disease, with extensive cerebral infarction areas. In the $5^{\text {th }}$ post-operative day, the patient's condition evolved with multiple organ dysfunction and cerebral stroke with exitus (Figure 5).

Figure 5 A,B. 4th post-operative day - the axial and coronal slices CT scan revealed the absence of the medial wall of the right maxillary sinus, right inferior and medial turbinates; $\mathbf{C}$. Axial plain $\mathrm{CT}$ indicating a hypodense area in the right cerebral fossa suggestive of cerebral stroke.

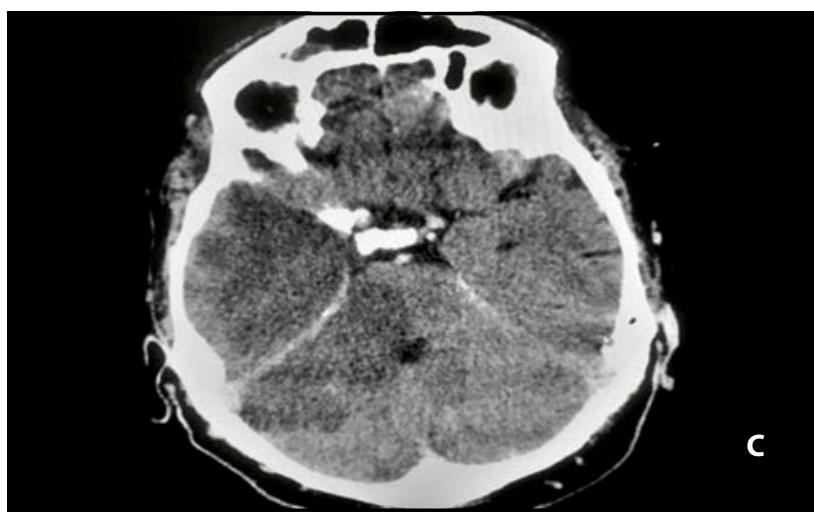



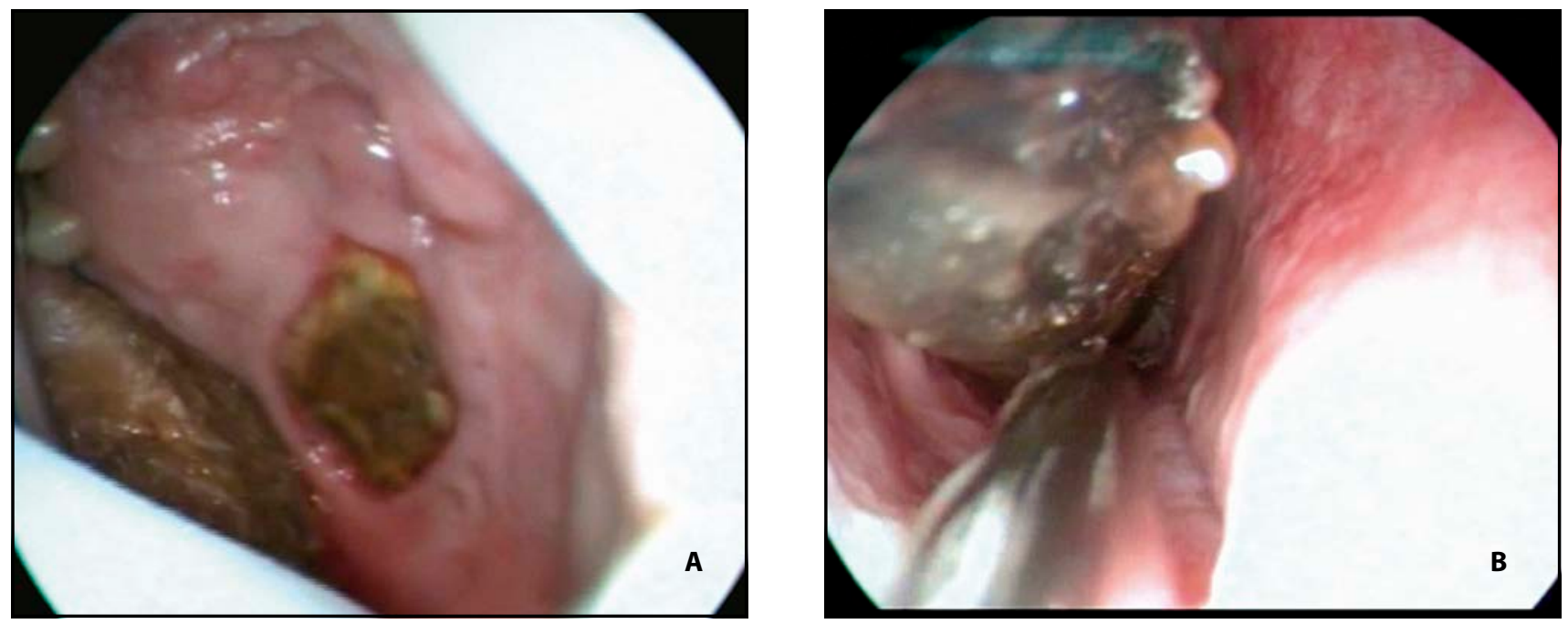

Figure 6 Black eschar over the hard palate $(\mathbf{A})$ and over the right inferior turbinate $(\mathbf{B})$.

\section{CASE 2}

An 18-year-old male patient presented in our ENT Clinic with pain and swelling on the right side of the face, diplopia, bilateral nasal obstruction and fever $\left(39^{\circ} \mathrm{C}\right)$ for $48 \mathrm{~h}$. He had a past history of acute myeloid leukemia treated with chemotherapy and allotransplantation of stem cells six months prior. Two months before this presentation he developed graft versus host dis-
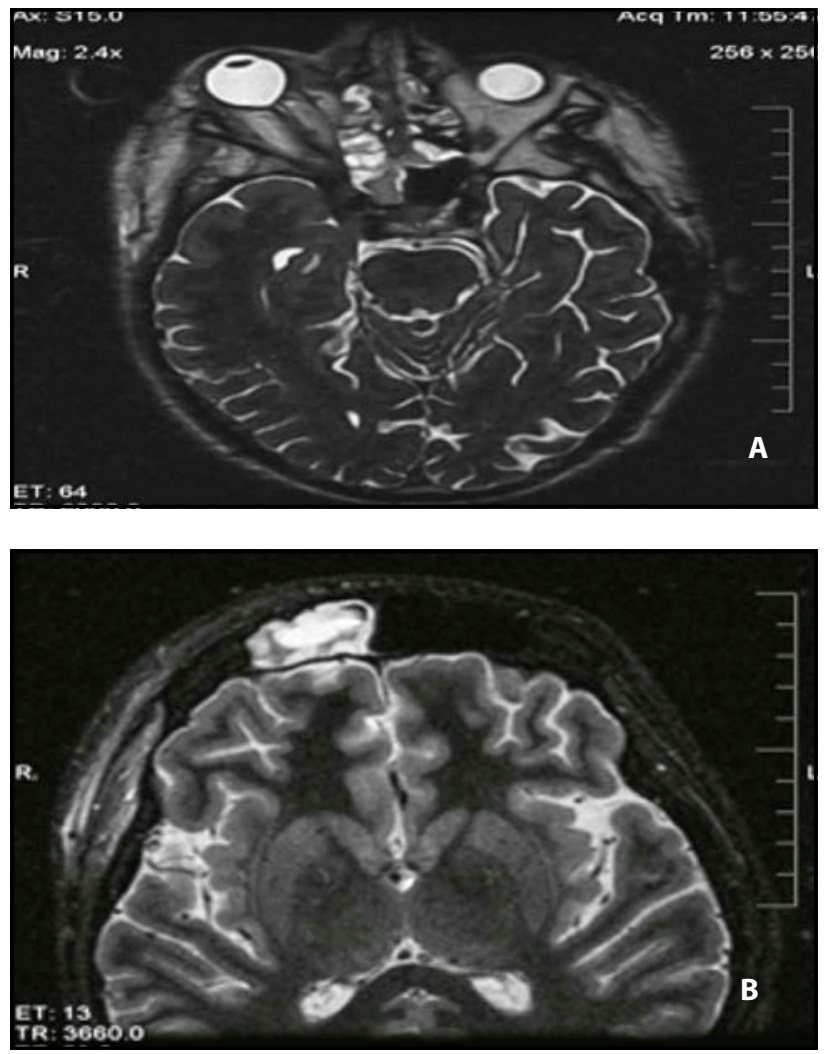

ease for which he received treatment with SoluMedrol $2 \mathrm{mg} / \mathrm{kg}$ with complete remission of symptomatology.

The ENT clinical examination showed right facial oedema, palpebral ptosis, necrotic crust on the hard palate and right nasal passage (Figure 6). The ophthalmologic examination revealed impairment of cranial nerves I, III, V.

The laboratory exams revealed a normal level of leukocytes $(7300 / \mathrm{mmc})$, thrombocythemia $(34.000 / \mathrm{mmc})$, anaemia $(\mathrm{Hb} 9.9 \mathrm{~g} / \mathrm{dl})$, blood sugar of $503 \mathrm{mg} / \mathrm{dl}$, raised levels of inflammatory markers (CRP $202 \mathrm{mg}$, fibrinogen $891 \mathrm{mg}$ ). The culture of palatine lesion secretion was positive for Zygomycetes.

MRI of the paranasal sinuses suggested right maxillary, ethmoid, frontal and sphenoid sinus

Figure 7 A,B, C.. T2-weighted MR imaging in axial cut-off indicating contrast enhancement in the right ethmoid, frontal and sphenoid sinuses. The right orbital content was involved and there was no intracranial involvement.

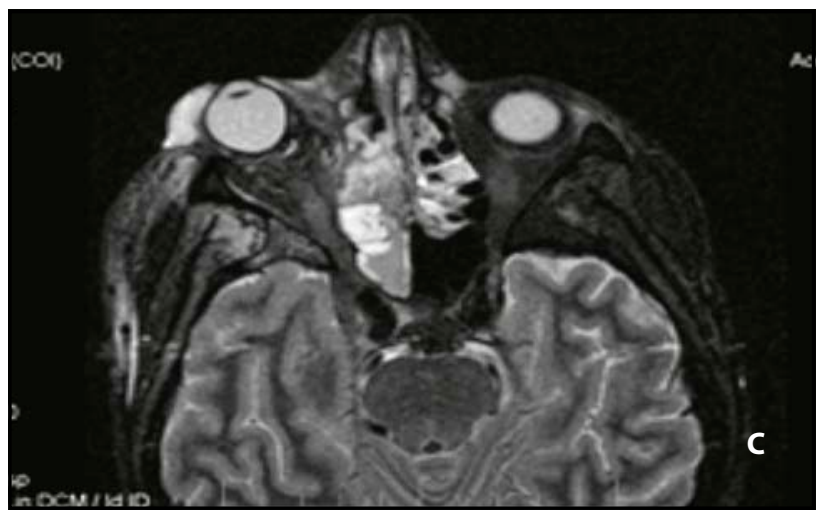



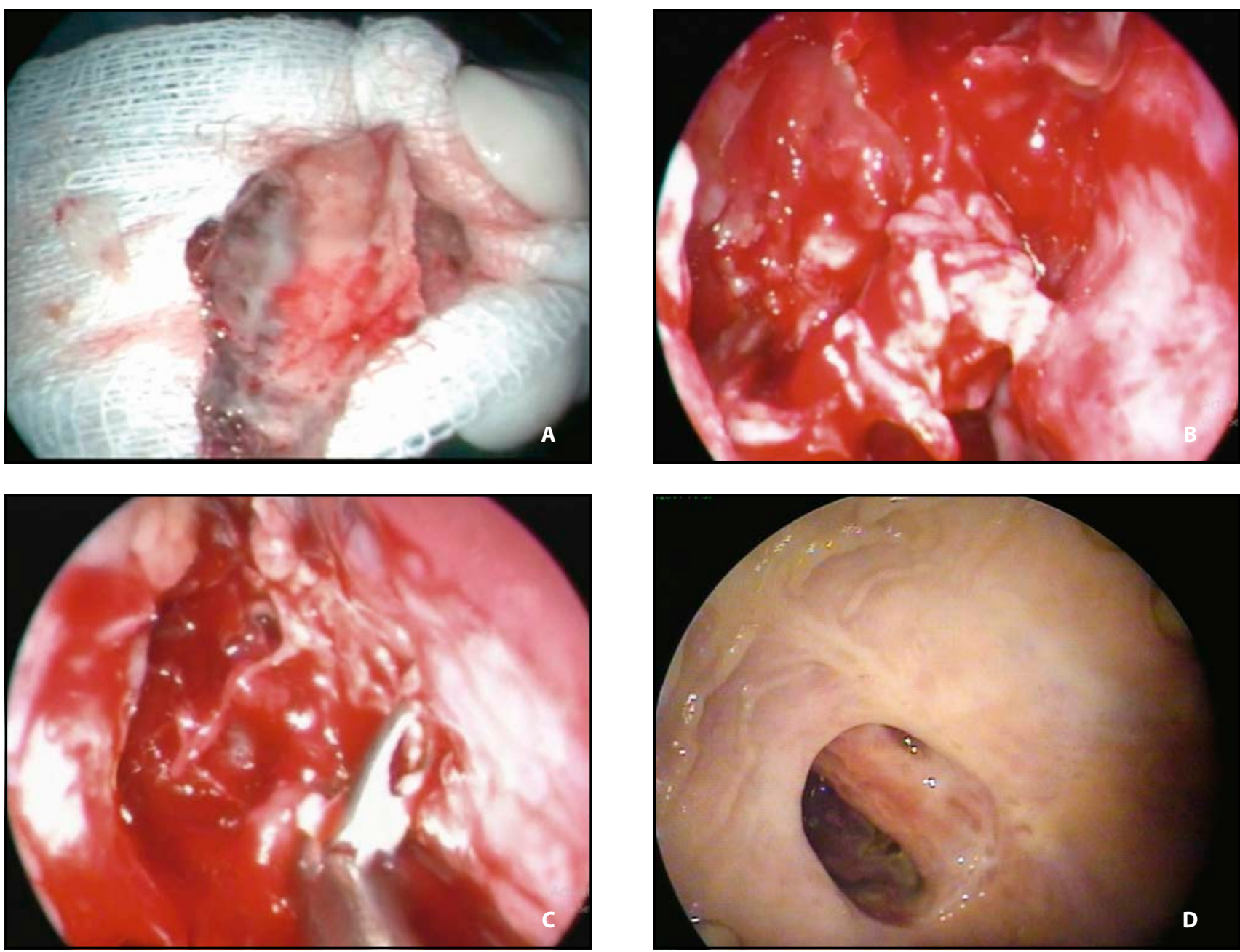

Figure 8 Intraoperative aspect $(\mathbf{A}, \mathbf{B}, \mathbf{C}) \mathbf{A}$. The right middle turbinate excised. B. The right maxillary sinus $\mathbf{C}$. The right ethmoid sinus $\mathbf{D}$. Postoperative aspect of the hard palate.

opacification with extension into the orbit (Figure 7).

The patient followed the same treatment protocol like the first patient - extended surgical debridement of the necrotic tissue (right medial maxillectomy, right ethmoidectomy, right sphenoidotomy and partial excision of the hard palate) combined with systemic antifungal treatment (Amphotericin B) with the improvement of the overall state (Figure 8). Due to the depletion of amphotericin B stock, the treatment was stopped at a total dose of $3250 \mathrm{mg}$.

The nuclear magnetic resonance on the $7^{\text {th }}$ day postoperatively revealed a lesion on the right orbital content which is why the patient was referred to the Department of Ophthalmology where he underwent right orbital exenteration (Figure 9).

Currently, at the 10-year follow-up, there is no evidence of disease recurrence and the patient remains asymptomatic (Figure 10).

\section{DISCUSSIONS}

In our cases, we opted for endoscopic surgical resection of the necrotic tissues. These case reports emphasize the importance of early diagnosis, appropriate medical and surgical therapy to obtain a significant survival rate in patients with this fatal disease. Although both patients followed the same treatment protocol, complete recovery was achieved in the second patient, whereas in the first one, due to late presentation, massive extension to the orbit, cavernous sinus and impossibility of total excision, the disease was complicated with multiple organ dysfunction and cerebral stroke.

In the first case, the diagnosis was delayed due to nonspecific symptomatology - severe headache during which the patient was investigated in another hospital unit. At the time the patient presented to our clinic, the disease was already in an advanced stage with hard palate, orbital and in- 


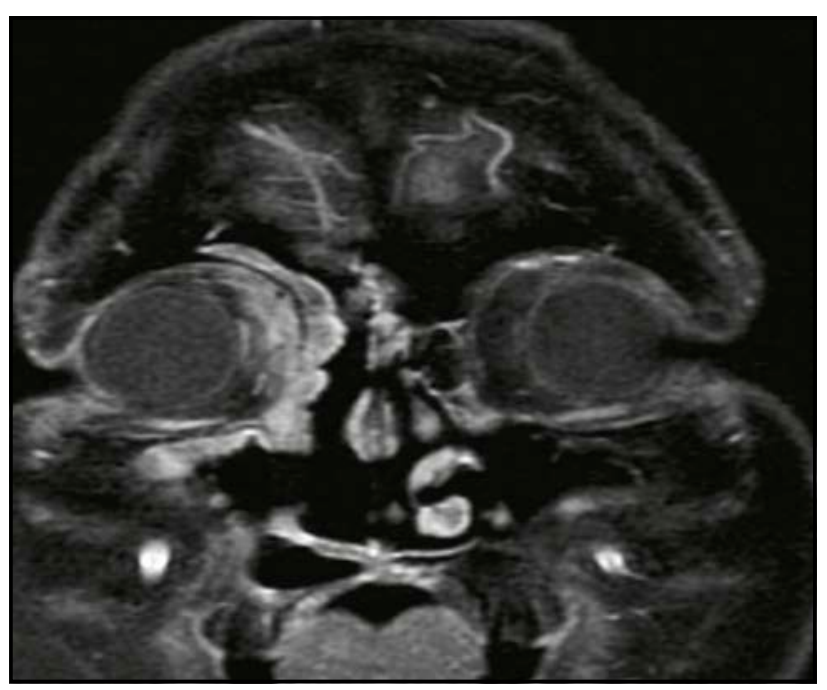

Figure 9 T2-weighted MRI in coronal view cut-off indicating hypertension of the periorbital soft tissue.

tracranial invasion. Once the cerebral area is involved, the treatment requires craniotomy with resection of all affected areas and administration of amphotericin B on all routes available: intravenously, interstitially or through cerebrospinal fluid perfusion ${ }^{17}$. In our case, because of the lack of a neurosurgery department, the radical debridement of all the affected tissue was impossible to be achieved.

In the second case, it was a question to determine if the patient should be exenterated or not. Until recently, the standard therapy for mucormycosis with orbital extension to prevent cerebral extension was orbital exenteration associated with aggressive surgical debridement and antifungal therapy ${ }^{18}$. Recently, studies have demonstrated that orbital exenteration is not required in all cases of orbital disease. The indication for exenteration depends on the patient's immune status, the severity of orbital extension, the aggressiveness of the disease, the response to the therapy and last but not least the judgment of the treating physician ${ }^{19}$. Roger Khon and Robert Hepler recounted from their experience eight cases of mucormycosis with orbital extension treated successfully without orbital exenteration. They described that the management included correction of risk factors, debridement of all necrotic tissues while ensuring an adequate sinus and orbital drainage, intravenous amphotericin $\mathrm{B}$ and daily irrigation and filling with amphotericin $\mathrm{B}$ the affected $\operatorname{areas}^{20}$. In our case, the nuclear magnetic resonance performed on the $7^{\text {th }}$ post-operative day suggested a lesion on the right orbital content, which is why the patient was referred to the department of ophthalmology where he underwent right orbital exen-

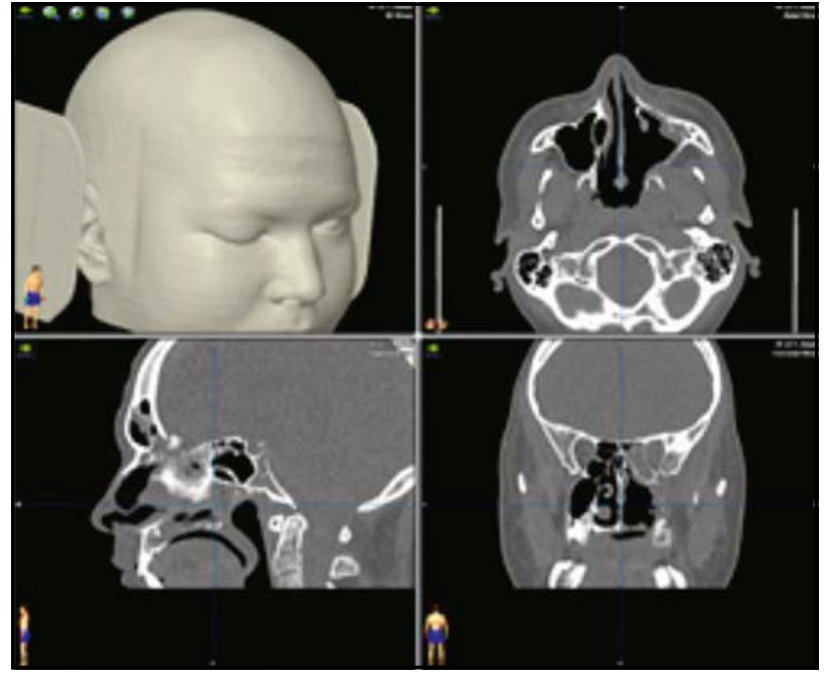

Figure 10 Neuronavigation imaging - 10-year postoperative aspect.

teration to prevent cerebral invasion.

A multidisciplinary approach with otolaryngology, neurosurgery, ophthalmology, intensive care, oromaxillofacial surgery and psychology is essential, because the surgical interventions can often be extensive and cosmetically disfiguring for patients.

The prognosis of mucormycosis is poor and it is influenced by the lesion extension to the noble tissues (the brain, the orbit, the cavernous sinus). An early aggressive approach of rhinosinusal mucormycosis has been shown to improve the survival rate ${ }^{21}$. Studies showed that the predictors of a low survival rate are: late diagnosis, neurological manifestations such as hemiplegia or hemiparesis, bilateral sinus involvement, treatment with iron chelating agents, renal disease and leukemia ${ }^{22}$. In a review of $170 \mathrm{pa}-$ tients made by Andrew Blitzer et al., the most reliable predictor of survival was the underlying disease $\mathrm{e}^{23}$. The major mortality factor is cerebral involvement with focal neurological deficits ${ }^{24}$.

By presenting these case reports, we would like to point out the importance of early diagnosis, which in our opinion represents the most important determinant of survival, the appropriate medical and surgical therapy to improve the outcomes of patients with this aggressive disease.

\section{CONCLUSIONS}

Mucormycosis is one of the most challenging pathologies in ENT because of the complications that may occur. It is an opportunistic infection affecting uncontrolled diabetics and immunocompromised individuals and has an aggressive nature, a tendency to recurrence and a high po- 
tential of invasiveness.

Unfortunately, there is no standard criteria for the diagnosis of mucormycosis. Diagnosis is based on detailed clinical examination, CT or MRI scan and confirmed by histopathological examination.

The diagnosis of mucormycosis should be established as quickly as possible in order to initiate the treatment for a favourable prognosis.

The elective treatment is extended surgical debridement of the necrotic tissue combined with systemic antifungal treatment (Amphotericin B) and reversal of the underlying risk factors.

Despite all these aggressive measures described, the prognostic remains poor.

Cerebral involvement carries a grave prognosis.

Conflict of interest: The authors have no conflict of interest.

Contribution of authors: All authors have equally contributed to this work.

\section{REFERENCES}

1. Kwon-Chung KJ. Taxonomy of Fungi Causing Mucormycosis and Entomophthoramycosis (Zygomycosis ) and Nomenclature of the Disease: Molecular Mycologic Perspectives. Clin Infect Dis. 2012;54(Suppl 1):S8S15. DOI: $10.1093 / \mathrm{cid} / \mathrm{cir} 864$.

2. Onyango J, Kayima JK, Owen WO. Rhinocerebral mucormycosis: case report. East African J. 2002;79(7):390-3.

3. Sahota R, Gambhir R, Anand S, Dixit A. Rhinocerebral Mucormycosis: Report of a Rare Case. Ethiop I Health Sci. 2017;27(1):85-90.

4. Prakash H, Chakrabarti A. Global epidemiology of mucormycosis. J Fungi. 2019;5(1):26. DOI: 10.3390/jof5010026.

5. Spellberg B, Edwards JJr, Ibrahim A. Novel perspectives on mucormycosis: pathophysiology, presentation, and management. Clin Microbiol Rev. 2005;18(3):556-69.

6. Wali U, Balkhair A, Al-Mujaini A. Cerebro-rhino orbital mucormycosis: an update. J Infect Public Health. 2012;5(2):116-26. DOI: 10.1016/j. jiph.2012.01.003. Epub 2012 Mar 27.

7. Alleyne CH Jr, Vishteh AG, Spetzler RF, Detwiler PE. Long-terme survival of a patient with invasive cranial base rhinocerebral mucormycosis treated with combined endovascular, surgical and medical therapies: case report. Neurosurg. 1999;45(6):1461-3; discussion 1463-4.

8. Ohtomo K, Ueta T, Nagahara M. Traumatic orbital apex syndrome. Can J Ophthalmol. 2015;50:e7-e8. DOI: 10.1016/j.jcjo.2014.10.012.
9. Petrikkos G, Skiada A, Lortholary O, Roilides E, Walsh TJ, Kontoyiannis DP. Epidemiology and Clinical Manifestations of Mucormycosis. Clin Infect Dis. 2012;54(Suppl 1):S23-34. DOI: 10.1093/cid/cir866.

10. Silvernan CS, Mancuso AA. Periantral soft-tissue infiltration and its relevance to the early detection of invasive fungal sinusitis: CT and MR findings. AJNR Am J Neuroradiol. 1998;19(2):321-5.

11. Spellberg B, Walsh TJ, Kontoyiannis DP, Edwards J Jr, Ibrahim AS. Recent Advances in the Management of Mucormycosis: From Bench to Bedside. Clin Infect Dis. 2009;48(12):1743-51. DOI: 10.1086/599105.

12. Blyth CC, Gomes L, Sorrell TC, da Cruz M, Sud A, Chen SC. Skull-base osteomyelitis: fungal vs. bacterial infection. Clin Microbiol Infect. 2011;17(2):306-11. DOI: 10.1111/j.1469-0691.2010.03231.x.

13. deShazo RD, O’Brein M, Chapin K, Soto-Aguilar M, Gardner L, Swain R. A new classification and Diagnostic Criteria for Invasive fungal sinusitis. Arch Otolaryngol Head Neck Surg. 1997;123(11):1181-8.

14. Alobid I, Bernal M, Calvo C, Vilaseca I, Berenguer J, Alos L. Treatment of Rhinocerebral Mucormycosis by Combination of Endoscopic Sinus Debridement and Amphotericin B. Am I Rhinol. 2001;15(5):327-31.

15. Spellberg B., Ibrahim A. Recent Advances in the Treatment of Mucormycosis. Curr Infect Dis Rep. 2010;12(6):423-9. DOI: 10.1007/s11908-0100129-9.

16. Katragkou A, Walsh TJ, Roilides E. Why is mucormycosis more difficult to cure than more common mycoses? Clin Microbiol Infect. 2014;20 Suppl 6:74-81. DOI: 10.1111/1469-0691.12466. Epub 2014 Jan 28.

17. Adler DE, Milhorat TH, Miller JI. Treatment of rhinocerebral mucormycoosis with i.v., interstitial and cerebrospinal fluid administration of amphotericin B: case report. Neurosurgery. 1998;42(3):644-8; discussion 648-9.

18. Ferry AP. Abedi S. Diagnosis and management of rhinoorbitocerebral and mucormycosis (phycomycosis): A report of 16 personally observed cases. Ophthalmology. 1983;90(9):1096-104.

19. Songu M, Unlu HH, Gunhan K, Ilker SS, Nese N. Orbital exenteration: A dilemma in mucormycosis presented with orbital apex syndrome. Am J Rhinol. 2008;22(1):98-103. DOI: 10.2500/ajr.2008.22.3121.

20. Kohn R, Hepler R. Manage ment of Limited Rhino-or bital Mucormycosis without Exenteration. Ophthalmology. 1985;92(10):1440-4.

21. Palejwala SK, Zangeneh TT, Goldstein SA, Lemole GM. An aggressive multidisciplinary approach reduces mortality in rhinocerebral mucormycosis. Surg Neurol Int. 2016;7:61. DOI: 10.4103/2151-7806.182964. eCollection 2016.

22. Yohai RA, Bullock JD, Aziz AA, Markert RJ. Survival Factors in RhinoOrbital-Cerebral mucormycois. Surv Opththalmol. 1994;39(1):3-22. DOI: 10.1016/S0039-6257(05)80041-4.

23. Blitzer A, Lawson W, Meyers BR, Biller HF. Patient survival factors in paranasal sinus mucormycosis. Laryngoscope. 1980;90(4):635-48.

24. Jayalakshmi S, Reddy RG, Borgohain R, Subramanyam C, Panigrahi M, Sundaram C, et al. Predictors of mortality in rhinocerebral mycosis. Neurol India. 2007;55(3):292-7. 